\title{
Inhibition of HIV-1 fusion with small interfering RNAs targeting the chemokine coreceptor CXCR4
}

\author{
N Zhou' ${ }^{1}$ J Fang ${ }^{1}$, M Mukhtar, E Acheampong and RJ Pomerantz \\ The Dorrance H Hamilton Laboratories, Center for Human Virology and Biodefense, Division of Infectious Diseases and Environmental \\ Medicine, Department of Medicine, Jefferson Medical College, Thomas Jefferson University, Philadelphia, PA, USA
}

\begin{abstract}
RNA interference (RNAi) is an evolutionarily conserved process by which plants and animals protect their genomes utilizing small, double-stranded RNAs to degrade target RNAs in a sequence-specific manner. Post-transcriptional gene silencing by these moieties can lead to degradation of both cellular and viral RNAs. It has recently been shown that double-stranded, small interfering RNAs (siRNAs) of 21-25 nucleotides can be transfected into relevant cells to target specific RNAs. This approach was utilized to inhibit human immunodeficiency virus type I (HIV-1) infection in human cells. siRNAs with homology to a motif in the mRNA that encodes for the HIV-1 chemokine coreceptor CXCR4 was utilized. Complementary studies via immunofluorescence microscopy and fluorescence-activated cell sorting demonstrated downregulation of CXCR4 from the surface of cells transfected with the specific siRNAs. As well, siRNAs without sequence homology to CXCR4 were used as controls and demonstrated no downregulation of CXCR4. siRNAs targeted to another chemokine coreceptor, APJ, showed specificity for downregulation of APJ but had no effects on CXCR4.
\end{abstract}

Transfections with siRNAs targeting CXCR4 mRNA were shown to inhibit HIV-1 envelope fusion, which is relatively resistant to most viral inhibitors targeting chemokine coreceptors. The specificity of this effect was demonstrated by the inhibition of fusion by CXCR4-tropic and dual-tropic (CXCR4 and CCR5) envelope glycoproteins from HIV-1 on CXCR4+ indicator cells, but the lack of effects by siRNAs targeting CXCR4 mRNA on dual-tropic HIV-1 envelopes in CCR5+ indicator cells utilizing these fusion assays. Interestingly, siRNAs targeting CXCR4 selectively inhibited CXCR4tropic cell-free virus infection of human cells but at only modest levels as compared to cell:cell fusion. siRNA may be a potential molecular therapeutic approach to alter a cellular cofactor critical for infection of human cells by relevant strains of HIV-1. The targeting of a cellular cofactor, rather than the HIV-1-specific mRNAs or genomic RNA, holds promise as the rapid mutational ability of the HIV-1 genome may obviate the potential clinical use of RNAi directly against this virus. Gene Therapy (2004) 11, 1703-1712. doi:10.1038/ sj.gt.3302339; Published online 12 August 2004

Keywords: siRNA; HIV-1; CXCR4; RNA interference; chemokine receptor; fusion

\section{Introduction}

Recent studies have shown that diverse 'micro-RNAs' of approximately 22-25 nucleotides are critical in animal development, including mammals. ${ }^{1-5}$ Small temporal RNAs have been well studied in the control of precise developmental events in nematodes. ${ }^{6}$ Of note, noncoding RNAs make up $98 \%$ of the genome in humans and are now known to act as critical factors in complex regulation cascades of all eukaryotic cells. ${ }^{7,8}$ As such, these small RNAs have been undergoing extensive investigations for use in understanding the function of multiple cellular proteins as well as potential antimicrobial therapy against various intracellular pathogens. ${ }^{9-17}$

Originally discovered by accident in studies of Caenorhabditis elegans, double-stranded RNAs were also

Correspondence: RJ Pomerantz, The Dorrance H Hamilton Laboratories, Center for Human Virology and Biodefense, Division of Infectious Diseases and Environmental Medicine, Department of Medicine, Jefferson Medical College, Thomas Jefferson University, Philadelphia, Pennsylvania 19107, USA

${ }^{1}$ These two authors contributed equally to this work

Received 11 August 2003; accepted 9 April 2004; published online 12 August 2004 shown to alter inheritable characteristics in these organisms. ${ }^{4}$ This was quickly followed by demonstration of post-transcriptional gene silencing (PTGS) by these moieties in plants and then other animals, and is now commonly referred to as RNA interference (RNAi). ${ }^{14}$ It has been clearly demonstrated that double-stranded RNA, with homology to a specific gene sequence, can act as a 'trigger' for PTGS. Unique aspects of this process are both the sequence specificity, leading to targeted mRNA degradation, and the requirement that of small interfering double-stranded RNAs (siRNAs) 21-25 nucleotides be produced via cleavage of longer double-stranded RNAs, proceeding to ongoing sequence-specific attack of target RNAs. ${ }^{18}$ These studies have demonstrated that this mechanism does not involve interferon, as longer (ie greater than 30 nucleotides) double-stranded RNAs would induce. Unlike RNAi, interferons lead to global, nonspecific effects on many cellular and viral RNAs. ${ }^{3}$ The cleavage of targeted RNAs in RNAi occurs via RNase III-like enzymes, entitled 'Dicer'. The siRNAs that are produced function in an siRNA protein complex or RNA-induced silencing complex (RISC), containing ATP and a helicase for unwinding of siRNA strands. In certain non-mammalian systems, it has been clearly shown that single-stranded siRNAs bind to the target 
mRNA and then lead to the formation of doublestranded RNAs by RNA-dependent RNA polymerase (RdRP). The new double-stranded RNAs are then cleaved by Dicer into further 21-25-nucleotide siRNAs. This last step acts as an amplification or catalytic process, which increases the intracellular efficiency of RNAi. ${ }^{19,20}$ Of note, though, this amplification step has not been demonstrated in mammals, and recent initial data suggest that it may not take place in either mammals or Drosophila. ${ }^{21}$

siRNAs, which seem to always require short $3^{\prime}$ overhangs, 3,22 were first shown to be useful in altering gene product expression by transfection into invertebrates. These siRNAs can be chemically synthesized or created via in vitro transcription systems, and maintain their activity after introduction into mammalian cells. ${ }^{2,3}$ Recent studies also suggest that siRNAs may be quite active in mammals when introduced in vivo. ${ }^{15}$

Of importance, the primary function of some of these evolutionarily conserved double-stranded RNAs may be in the protection of the host genome from transposons and viruses. ${ }^{2,16,17}$ The first demonstrations that RNAi acted against viruses were performed in plant systems and then recently in Drosophila. ${ }^{23}$ Based on these studies, it has become clear that siRNAs maybe useful in developing molecular therapeutics for human viral pathogens.

Of note, human immunodeficiency virus type I (HIV1) is a possible target for development of RNAi to halt initial infection of human cells or spread of the virus after infection, with the ability to target multiple aspects of its complex life cycle. ${ }^{13,24}$ One potential point of attack using this newly developed technology would be the site of virion binding and entry of critical human target cells.

HIV-1 enters most human cells by initial binding to the high-affinity CD4 receptor, as well as binding of the envelope glycoprotein (gp120) to critical chemokine coreceptors, especially CXCR4 and CCR5. ${ }^{25,26}$ CXCR4 belongs to the family of seven transmembrane G-proteincoupled receptors. ${ }^{27-31}$ These coreceptors transduce signals via heterotrimeric G-proteins. CXCR4 has been clearly identified as one of the major coreceptors for HIV1 cell entry in vivo. ${ }^{26}$ As well, recent studies with knockout mice of CXCR4 have demonstrated that this molecule plays an important role in immunomodulation, organogenesis, hematopoiesis and cerebellar neuron migration. $^{32-34}$

The discovery of chemokine coreceptors for HIV-1 tropism demonstrates the phenotypic diversity conferred by coreceptor specificity. Viral isolates obtained in HIV-1infected individuals during asymptomatic stages generally are CCR5-tropic. ${ }^{35}$ In contrast, a majority of isolates derived after progression of disease are CXCR4-tropic. As well, it has been reported that viral isolates derived from transition of HIV-1-infected nonprogressors to progressors are dual-tropic (CCR5 and CXCR4). ${ }^{35}$ It has also been demonstrated that CXCR4-tropic phenotype of recombinant HIV-1 strains and primary HIV-1 isolates are linked to a highly pathogenic phenotype in ex vivo lymphoid tissues, which is substantially inhibited by interfering with CXCR4-mediated entry. ${ }^{36}$ It was then demonstrated that the specificity of recombinant HIV-1 strains for CXCR4-tropism correlated with potent depletion of CD4+ T-lymphocytes in human lymphoid histocultures. ${ }^{37}$
Nevertheless, the development of moieties to inhibit or block interactions between the HIV-1 envelope and chemokine coreceptors is somewhat problematic. One of the problems in designing antichemokine coreceptor therapeutic agents is the complexity of the chemokine receptor-ligand interactions. ${ }^{31}$ This represents blocking of a tricomplex, gp120-CD4-CXCR4. The natural ligand of CXCR4 has been identified as stromal cell-derived cofactor (SDF-1), an alpha chemokine. SDF-1 $\alpha$ blocks the infection of T-lymphocytes with CXCR4-tropic strains of HIV-1. ${ }^{27}$ Based on these data, molecular therapeutics in attempts to downregulate CXCR4 expression on the surface of relevant target cells have become an area of active investigation. As approximately $50 \%$ or more of individuals infected with HIV-1 convert from CCR5 to CXCR4-tropic viral strains during progression to the acquired immune deficiency syndrome (AIDS), ${ }^{35}$ we hypothesized that CXCR4 would be a potentially important target for significant downmodulation utilizing newly developed RNAi technologies.

In this report, it is demonstrated that siRNAs specific to CXCR4 mRNA can downregulate this chemokine coreceptor's expression on the surface of target cells. As well, the siRNA negatively affects HIV-1 fusion with CXCR4-tropic viral envelopes.

\section{Results}

\section{Suppression of CXCR4 and APJ expression} on cell surfaces by siRNA

To analyze the ability of CXCR4 siRNA to suppress specifically CXCR4 expression, CXCR4-GFP (GFP: green fluorescent protein) or APJ-GFP expression vectors and siRNAs were cotransfected into HOSCD4 cells. As shown as Figure 1a, $48 \mathrm{~h}$ after transient transfection, GFP siRNAs efficiently decreased expression of both fusion proteins, CXCR4-GFP and APJ-GFP, while CXCR4 siRNA only reduced CXCR4-GFP expression. Cotransfection with APJ-GFP and CXCR4 siRNA had no effect on expression of APJ-GFP. A $2 \mathrm{nM}$ concentration of siRNAs was used in these studies, as inhibition of CXCR4 expression dropped off sharply below this input concentration and no further clear decreases in CXCR4 levels were noted with increased levels of siRNA input. Of note, no cellular toxicity was noted in transfections using these siRNAs (not illustrated).

We then evaluated siRNA specificity on target gene expression in stable cell lines. The results illustrated in Figure 1b demonstrate that GFP siRNA efficiently downregulated APJ-GFP expression in HOS cells stably expressing CD4 and APJ-GFP, while CXCR4 siRNA had no effect on APJ-GFP expression in these cells, as assayed via immunofluorescence microscopy. Extended experiments were carried out to determine whether siRNAs suppress specifically CXCR4 and APJ expression, in stable 293 cell lines expressing CXCR4-GFP and APJGFP, respectively. The data in Figure 1c demonstrate that, in stably transfected 293 cell lines, CXCR4-GFP was dramatically downregulated by transfection of CXCR4 siRNA but not by APJ siRNA. Of note, conversely, APJGFP expression was suppressed only by APJ siRNA but not by CXCR4 siRNA. As such, these data suggest that GFP fusion proteins with relevant chemokine corecep- 
a
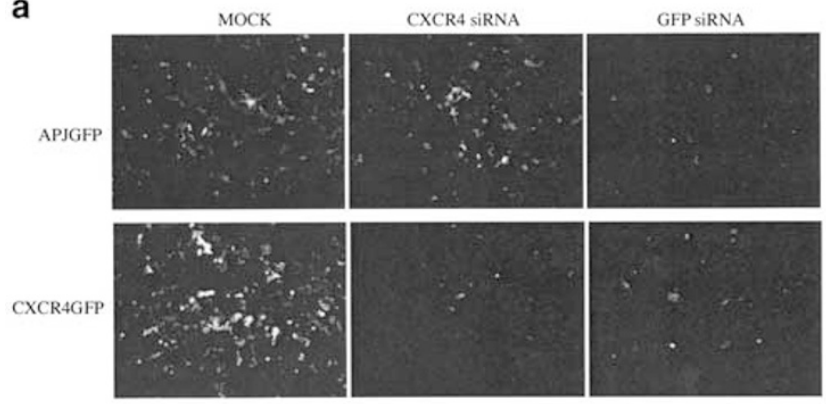

b
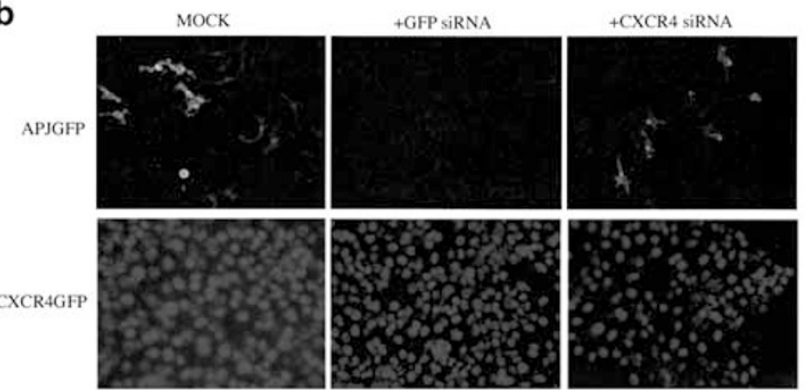

CXCR4GFP

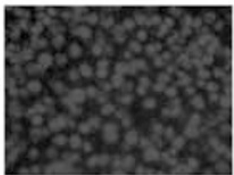

C

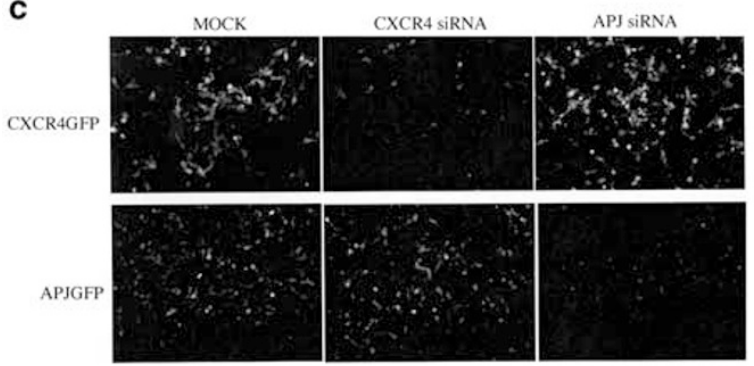

Figure 1 Specificity of the effects of CXCR4 siRNA on target gene expression. The HOS CD4 cell line was cotransfected with constructs expressing APJ-GFP or CXCR4-GFP fusion proteins and $2 n M$ of CXCR4 siRNA or GFP siRNA (a). The stable cell line HOS CD4 expressing APJGFP (b) and the 293 cell lines expressing CXCR4-GFP or APJ-GFP (c) were transfected with $2 n M C X C R 4$ siRNA or APJ siRNA. The cells were fixed $48 \mathrm{~h}$ later and analyzed by fluorescence microscopy. The lower panels in (b) demonstrate cell numbers by $4^{\prime}, 6^{\prime}$-diamidino-2-phenylindole hydrochloride (DAPI) staining of the nuclei (blue). The results shown are representative of at least two independent experiments.

tors can be downregulated by siRNAs, and thus function as a initial 'proof of concept' for this approach.

\section{Downregulation of CXCR4 expression}

A full series of HOS indicator cells were then transfected with siRNA specific to CXCR4 to quantify the siRNAs' potency in downregulating CXCR4. siRNA specific to GFP as a target was used as a negative control. As noted above, the specificity of GFP siRNA was confirmed previously in HOSCD4APJ-GFP cells (Figure 1a and b). We then sought to demonstrate the specificity of CXCR4 siRNA. As shown in Figure 2, in HOSCD4CXCR4 cells, CXCR4 was downregulated with at least $80 \%$ efficiency by CXCR4 siRNA, but only minimal decreases in CXCR4+ cells were noted with GFP siRNA, as analyzed by FACS. CCR5 expression revealed no change in the HOSCD4CCR5 indicator cells, with either siRNA moiety. Thus, these results provided evidence that CXCR4
siRNA specifically inhibits CXCR4 expression on cell surfaces, although full and absolute downregulation of CXCR4 was not obtained with transfected siRNAs.

\section{Activities of CXCR4 siRNA in blocking CXCR4-mediated cell membrane fusion}

We then utilized a well-characterized, luciferase-based cell-cell fusion assay to evaluate the abilities of CXCR4 siRNA in blocking CXCR4- and HIV-1 gp120-mediated cell membrane fusion. As effector cells, 293 cells were coinfected with vaccinia virus-encoded HIV-1 gp120 and vaccinia virus-encoded luciferase under the control of the T7 promoter. This partciular target cell system was chosen as it lends itself to clear quantitation of HIV-1 envelope-induced cell fusion. ${ }^{38}$ HOSCD4CXCR4 target cells were cotransfected with T7 RNA polymerase and CXCR4 siRNA. The results and relative percentages of changes in fusion activity are presented in Figure 3. Transfection of CXCR4 siRNA resulted in approximately 65 and 75\% inhibition of CXCR4 coreceptor activity for both IIIB (CXCR4-tropic, Figure 3a; $P \leqslant 0.003$ by Student's t-test) and 89.6 (dual-tropic (CXCR4 and CCR5, Figure $3 \mathrm{~b} ; P \leqslant 0.003)$ ) HIV-1 isolates, respectively. No blocking of CCR5 coreceptor activity was noted with the CXCR4 siRNA (Figure 3c; $P=0.63$ (NS)), and GFP siRNA, as negative control, had no inhibitory effects on CXCR4mediated cell fusion (Figure 3). As such, these data demonstrate a selective inhibitory effect on HIV-1induced cell fusion by siRNA to CXCR4. In vitro fusion assays are known as usually difficult systems in which to demonstrate inhibitory effects with other anti-CXCR4 moieties. ${ }^{39}$ Of note, though, in this system we reported previously that a specific CXCR4 inhibitor, ALX40-4C, inhibited over $80 \%$ of cell:cell fusion at micromolar concentrations. ${ }^{38}$ These are interesting findings, as cellto-cell fusion is likely a critical mechanism of HIV-1 spread within lymphoid tissues of infected individuals.

\section{Inhibition of HIV-1 production by CXCR4 SiRNA analyzed using cell-free viral infections}

The effects of downregulated CXCR4 by siRNA were also evaluated using the cell-free HIV-1 T-tropic (CXCR4) strain NL4-3 and M-tropic (CCR5) strain ADA to infect the HOS cell-line series. No toxicity on cell proliferation was detected with these siRNAs (not illustrated). As illustrated in Figure $4 a$, inhibition of viral infection using a CXCR4-tropic virus (ie NL4-3) was demonstrated in CXCR4+ cells using the CXCR4 siRNA $(P \leqslant 0.03)$, but the inhibition was relatively modest. The control siGFP had little effect on viral growth in these cells. Finally, the siRNAs did not alter R5-tropic viral growth on CCR5+ cells (Figure $4 \mathrm{~b}$; all $P$-values were not sigificant). Of interest, though, siRNA's effects on CXCR4 was more robust in inhibiting HIV-1 fusion (Figure 3), as compared to cell-free virion infectivity. This divergence in effects of cell-free virus infection versus cell:cell fusion, with efficient but not total downregulation of CXCR4, is important as it can be hypothesized to be secondary mechanistically to differential utilization of low levels of CXCR4 surface densities required for these viral processes.

To further analyze the effects of downregulation of CXCR4 by siRNA on cell-free viral infection, a complementary system was utilized in which luciferase-expres- 
Anti-CXCR4
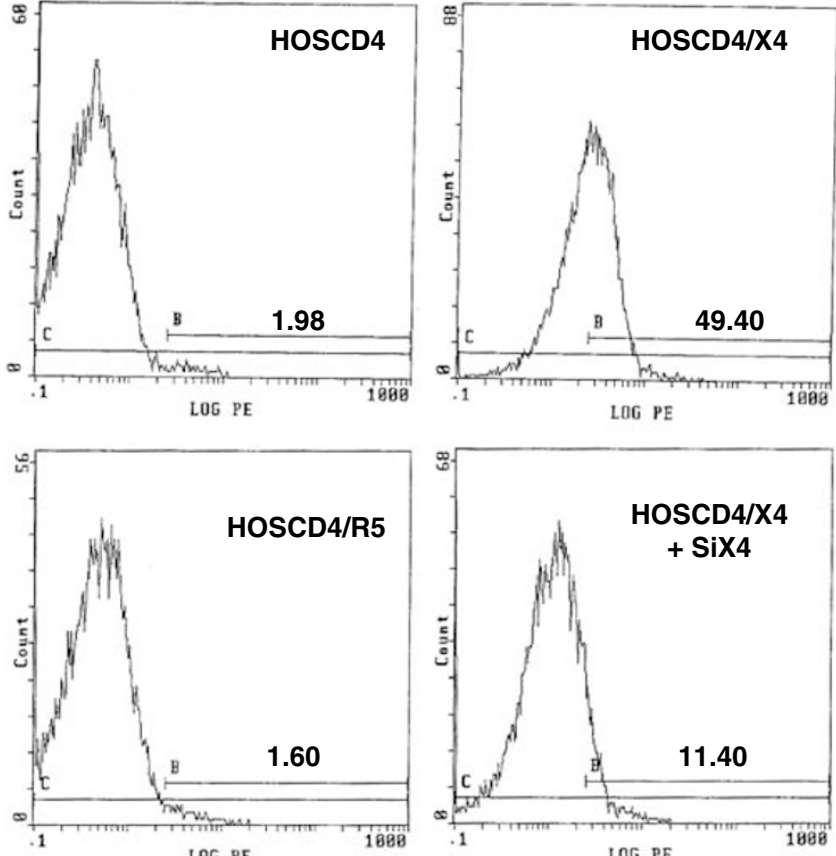

LOG PE
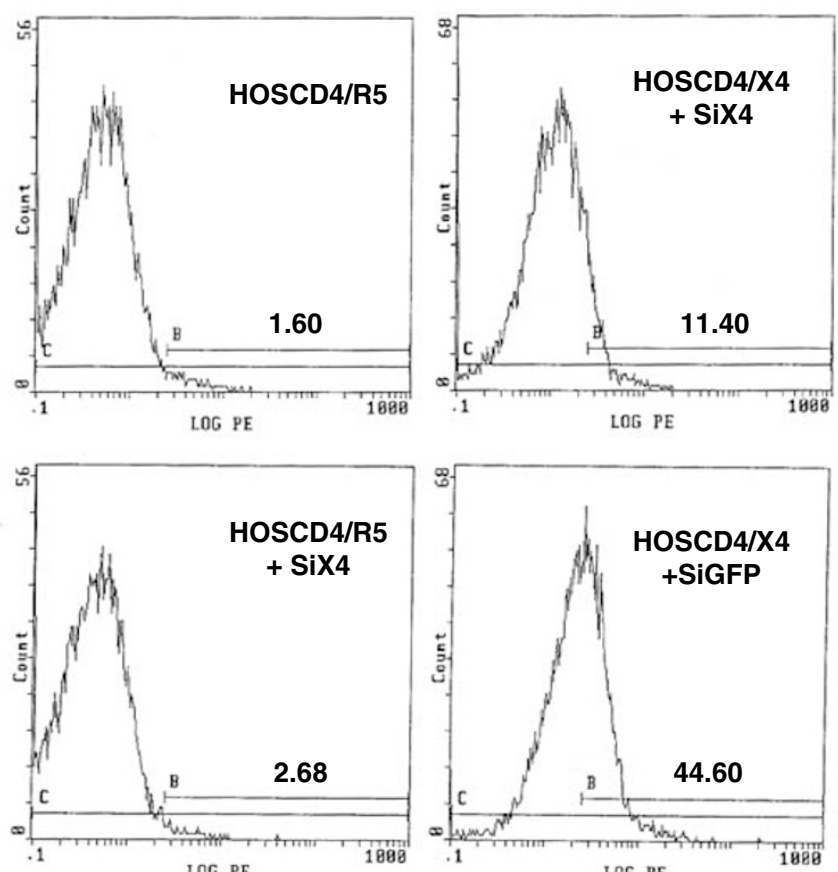

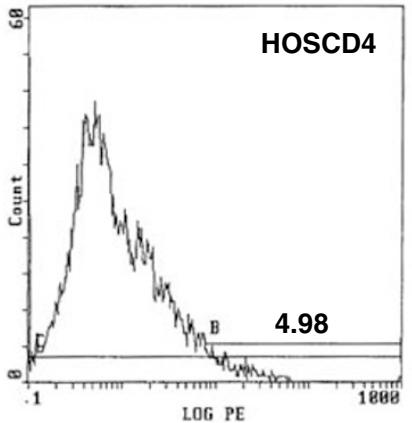

Anti-CCR5
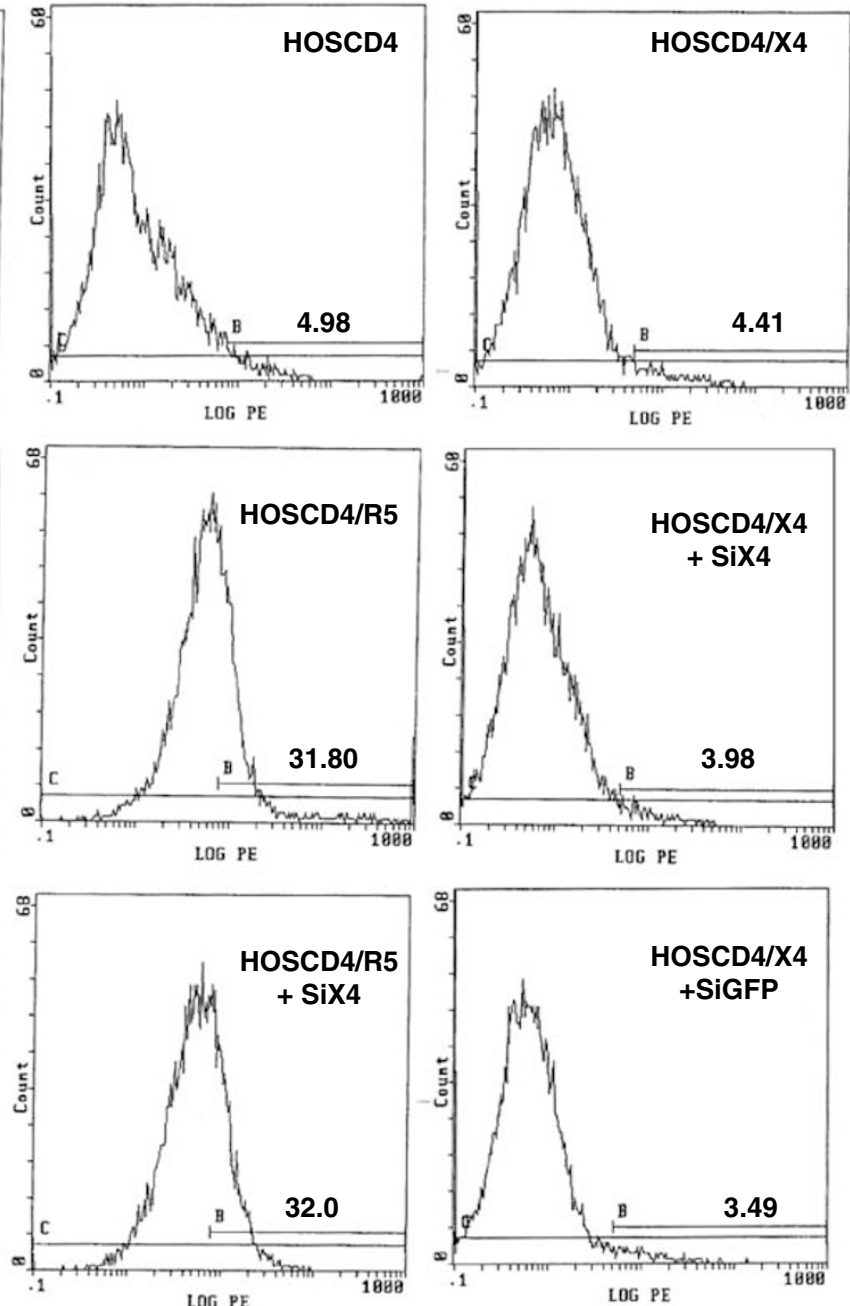

Figure 2 Suppressed CXCR4 expression by CXCR4 siRNA. The HOS series of indicator cells (HOSCD4, HOSCD4/CXCR4 and HOSCD4/CCR5) were transfected or left untransfected with $2 \mathrm{nM}$ of CXCR4 siRNA or GFP siRNA in each well of six-well plates, as indicated in the panels. Cells were harvested $48 \mathrm{~h}$ later and analyzed by fluorescence-activated cell sorting (FACS). The cells were stained by using mouse anti-human CXCR4 or CCR5 as an initial antibody (noted at the top of the figure), followed by PE-conjugated anti-mouse IgG as a secondary antibody. The results shown are representative of at least two independent experiments. SiX4, siRNA to CXCR4; siGFP, siRNA to GFP.

sing reporter viruses were produced and used to infect U87MG cells (astrocytic glial cell lines), expressing CD4 alone, CD4/CXCR4 or CD4/CCR5. As expected, no obvious HXB2-luc growth was detected on CD4/CCR5expressing cells, but quite reasonable viral growth was demonstrated on CD4/CXCR4-expressing cells with a moderate decrease in cultures treated with CXCR4 siRNA (siCXCR4 versus siGFP- $P \leqslant 0.03$ (ANOVA analysis of variance: F-test); siCXCR4 versus nontransfected $-P \leqslant 0.004$; siGFP versus nontransfected $-P=0.11$ (NS)) (Figure 4c). These studies confirmed that CXCR4 siRNA could inhibit cell-free virus infection, as measured by luciferase activity, with X4-tropic reporter viruses, but less effectively than cell-cell fusion.

\section{Discussion}

This report demonstrates the ability of siRNAs to inhibit HIV-1-induced fusion, by targeting the mRNA for the
CXCR4 chemokine coreceptor. We used complementary approaches to demonstrate both CXCR4 surface downregulation after treatment with specific siRNAs and their activities in altering HIV-1 fusion. Of note, the specificity of this effect was demonstrated by the lack of inhibition of dual-tropic viral isolates in CCR5+ and CD4+ target cells, and the lack of downregulation of chemokine coreceptors other than CXCR4. As well, siRNA targeted to another chemokine coreceptor (ie APJ) had no nonspecific effects on CXCR4. Inhibition of cell-to-cell fusion via siRNA is especially promising, as it is difficult to efficiently demonstrate this effect using most chemokine coreceptor antagonists. ${ }^{39-42}$ Finally, inhibition of cell-free virion infection of CXCR4+ cells by CXCR4tropic virus was demonstrated with the RNAi approach, without inhibition of CCR5-tropic viruses on CCR5+ cells, but the potency was clearly lower than for cell-cell fusion.

RNAi has promise as a potent molecular therapeutic approach in attacking the life cycle of a wide variety of 
a

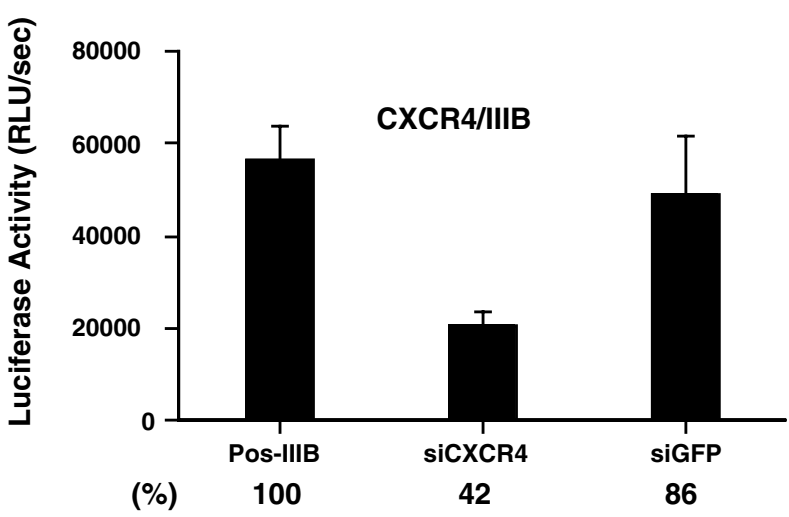

b

O্ঠ

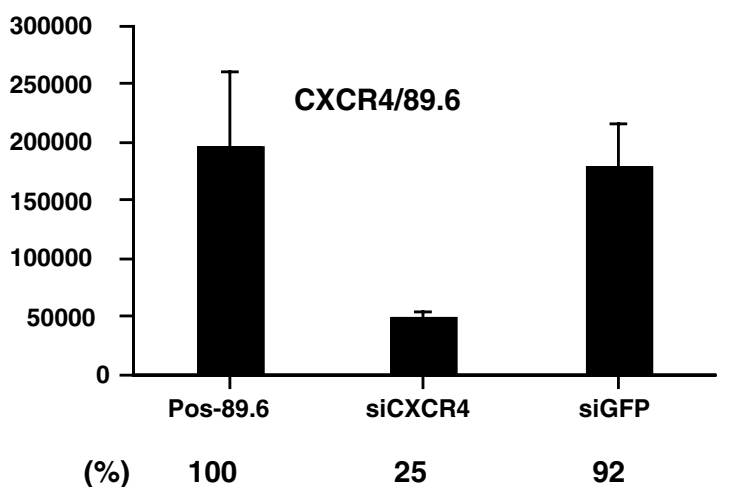

C

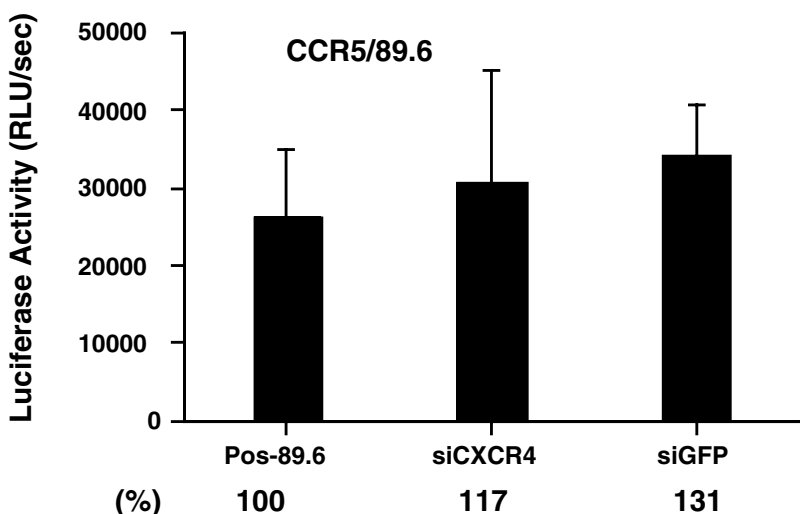

Figure 3 Effects of CXCR4 siRNA on cell-cell fusion mediated by CXCR4 and HIV-1 gp120. HOSCD4CXCR4 $(a, b)$ and HOSCD4CCR5 (c) target cells were cotransfected with siRNAs and plasmids encoding luciferase under the control of the T7 promoter. 293 effector cells were infected with a vaccinia virus encoding $T 7$ polymerase, and a vaccinia virus encoding strain-specific HIV-1 envelope proteins. Cell mixtures were maintained at $37^{\circ} \mathrm{C}$ for $4-6 \mathrm{~h}$. Fusion was determined by a luciferase assay. The numbers below each figure represent effects of siRNA on fusion activity as a percentage of the positive controls. Negative controls lacking HIV-1 envelopes are usually below $5 \%$ in this assay system. The values are the means \pm s.d. of quadruplicate samples, and the results are representative of two independent experiments.

human viral pathogens. Molecular therapy against HIV-1 has used both protein- and RNA-based modalities (for a review, see reference Dornburg and Pomrantz ${ }^{24}$ ). Previous RNA-based modalities, which have had some significant effects on HIV-1 replication in various model systems, include antisense, molecular decoys and ribozymes. The potential potency of RNAi may have importance as an antiviral agent, as compared to these other approaches. Previous studies have demonstrated siRNAs' inhibitory effects on polio virus, and recently human papilloma virus (HPV), hepatitis $\mathrm{C}$ virus (HCV) plus influenza. ${ }^{43-46}$

Effects on both cellular cofactor RNAs and HIV-1specific RNAs have also been demonstrated recently with siRNAs. ${ }^{47-52}$ An initial study has shown that CD4 mRNAs may be targeted by siRNAs as potential therapeutic interventions. ${ }^{51}$ One possible problem with this approach is the important detrimental immunomodulation of T-cell helper function, based on the robust depletion of the CD4 antigen on lymphoid cell surfaces. In addition, nuclear factor-kappa B (NF- $\kappa \mathrm{B})$, which has been demonstrated to be critical in HIV-1 transcriptional control, has also been targeted with RNAi with some success (ie approximately 40-50\% decrease in HIV-1 expression). ${ }^{52} \mathrm{~A}$ very recent study suggests that RNAi targeting CCR5 mRNA may be useful in inhibiting HIV-1 strains found relatively early in clinical disease (ie about $65 \%$ decrease in HIV-1 p24 antigen expression in these infectivity studies). ${ }^{53}$ Finally, both structural protein ( $g a g$ ) and regulatory protein (eg tat and rev) viral mRNAs have also been approached using siRNA inhibitors in relevant model systems in vitro. ${ }^{4-50}$

Of note, one report has demonstrated the potentially very important finding that siRNAs may gain entrance to the preintegration complex of HIV-1, leading to cleavage of the 'in-coming' viral genome. ${ }^{49}$ In addition, this same study demonstrated that siRNAs could be produced from hairpin motifs produced from transfected plasmids. This allows the constant or regulated intracellular expression of siRNAs, which are cleaved from the hairpin motifs produced in the plasmid-transfected cells. Of note, transfected siRNAs although potent in many scenarios have a relatively short inhibitory time course on their target RNAs, unless in situ amplification occurs with this process. ${ }^{47}$ As noted previously, it is not yet clear that RNAi amplification is a productive process in mammalian cells. As well, whether the RNAi effect spreads potently enough between cells to inhibit HIV-1 at a distance from the initial site of RNAi is not clear.

The CXCR4 coreceptor appears to be an important target for therapeutic intervention against HIV-1. A variety of antibodies, small molecular inhibitors and intracellular single-chain variable fragments (SFvs) have been used both in vitro, and some in vivo, to inhibit CXCR4-specific binding of HIV-1 in relevant target cells. $^{39-42,54-60}$ Of note, the in vivo-tested molecular inhibitors of CXCR4 did not appear to lead to significant adverse immunological effects. ${ }^{42}$ In addition, targeting cellular proteins that act as cofactors in the HIV-1 life cycle may be of greater reliability than targeting viral mRNA species or genomic RNA with RNAi, as the propensity of this virus to undergo rapid mutations would alter the long-term inhibitory capabilities of siRNAs directed against the virus itself.

In the present study, we have also developed an siRNA to the orphan G-coupled seven transmembrane receptor APJ. This unique coreceptor has been shown to bind and allow infection of cells by a number of HIV-1 strains. ${ }^{61-64}$ Its presence in the central nervous system 
(CNS) is of potential importance, ${ }^{64-66}$ and investigation of siRNAs' effects on this moiety is ongoing in our laboratories. CXCR4, as well, has been demonstrated on CNS-based cells. ${ }^{66,67}$ It has been recently demonstrated that RNAi also functions in mammalian neurons in vitro. ${ }^{68}$ As such, targeting CXCR4 and/or APJ in human CNS cells may be an important first step in targeting RNAi toward HIV-1-induced encephalopathy.

During preparation of this manuscript, an initial report suggested that siRNAs could be useful in

a

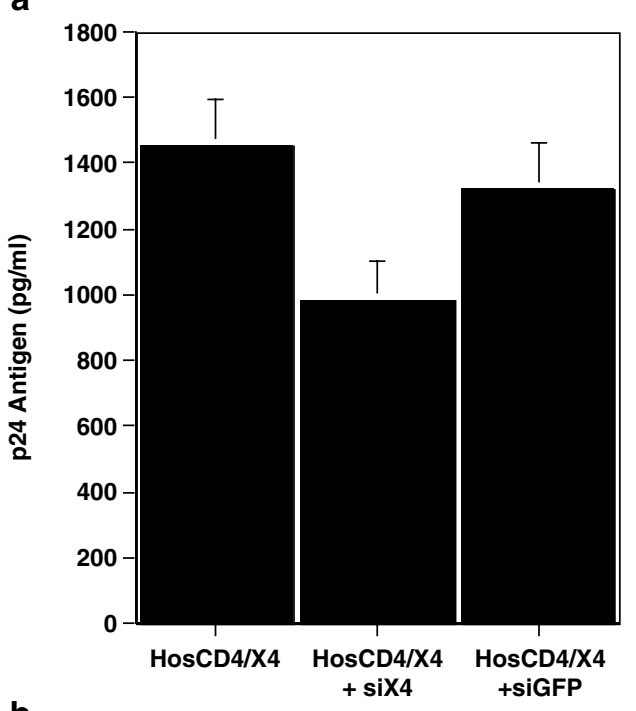

b

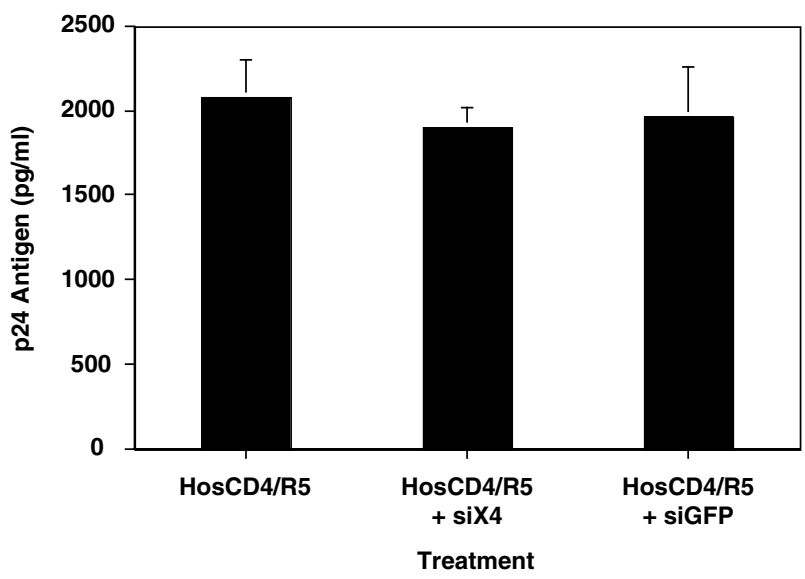

C

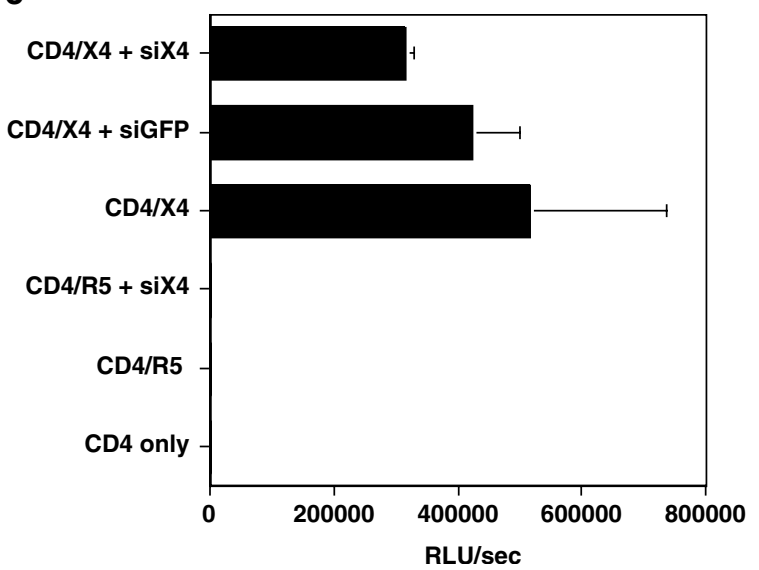

inhibiting both CCR5 and CXCR4 for intervening in HIV-1 infection. ${ }^{69}$ This relatively small study suggested that only a $60 \%$ decrease in CXCR4+ cells by siRNA led to very significant decreases in viral replication. It was surprising in this report that the level of viral production after only $48 \mathrm{~h}$ postinfection was above $150 \mathrm{ng} / \mathrm{ml}$ of p24 antigen equivalents in U87 indicator cells, even with zidovudine added after initial infection to prevent a second round of viral spread in these experiments. In addition, our present study is the first to demonstrate that siRNAs against CXCR4 can potently inhibit HIV-1mediated cell-to-cell fusion, a critical parameter altered by siRNA inhibition of CXCR4 activity, important for both intra- and interhost HIV-1 transmission in vivo, and not evaluated in the report by Martinez et al.

In our present study, inhibition of fusion by siRNA to CXCR4 was actually more robust than inhibition of cellfree virion infectivity, with the approximately $80 \%$ downregulation of CXCR4 that we achieved with this siRNA moiety. This is mechanistically interesting and may be secondary to differences in low chemokine coreceptor levels and densities on target cell surfaces required for these two processes. Of importance and paradoxically, previous studies of pharmacological drugs or small molecule inhibitors that target HIV-1 entry have shown them to be dramatically more potent at inhibiting cell-free viruses, as compared to cell:cell fusion. ${ }^{70}$ Of note, inhibition of CCR5-directed HIV-1 cell-to-cell fusion by siRNAs was also not analyzed in a recent study, $^{53}$ as only effects on infection with cell-free viruses were reported and their results were comparable to our findings. Although, in the present study, full downregulation of CXCR4 was not demonstrated using transfection approaches of siRNAs, certain modifications are possible in further model systems to increase efficiency. Future studies in primary T-lymphocytes of RNAi directed against CXCR4 will likely require transduction of these cells with retroviral vectors expressing hairpin motifs, which produce specific siRNAs within the cells. ${ }^{49}$ Inducible promoters will likely also be quite useful in these approaches.

In conclusion, these present studies demonstrate that RNAi can inhibit the expression of the critical HIV-1 chemokine coreceptor CXCR4. The utility of this approach toward inhibiting HIV-1 in peripheral blood and lymphoid tissue, as well as in possible reservoir or sanctuary sites such as the CNS, requires further investigation. Thus, the potential potency and utility of the RNAi approach to target multiple sites in the HIV-1

Figure 4 Inhibition of cell-free HIV-1 infection of CXCR4+ cells. The HOS cells, CD4/CXCR4 and CD4/CCR5, were used as targets for HIV-1 infection, $48 \mathrm{~h}$ after transfections with siRNAs targeting CXCR4 or GFP $m R N A s$. A $10 \mathrm{ng}$ portion of HIV-1 p24 antigen equivalents of viral strains (a) $\mathrm{NL}_{4-3}$ and (b) $A D A$ was utilized. After vigorous washing, viral outgrowth was assayed $72 \mathrm{~h}$ later, utilizing ELISA for HIV-1 p24 antigen expression. These data are from duplicate cultures with standard error bars, and are representative of two independent studies. (c) HIV-1 luciferase reporter virus infections. U87MG cells, expressing CD4 alone, CD4/CXCR4 or CD4/CCR5, were used as targets for HIV-1 ${ }_{H X B 2}$ luciferase reporter virus, $48 \mathrm{~h}$ after siRNA transfections $(2 \mathrm{nM})$. The cells, lysed $24 \mathrm{~h}$ after infection, were analyzed for luciferase activity (RLU/s) in a luminometer. As expected, the HIV-1 ADA luciferase reporter virus did not grow to significant levels on CD4/CXCR4+ cells (not illustrated). The data illustrated are from triplicate cultures with standard error bars, and are representative of two independent experiments. 
life cycle may allow clinically 'intracellular immunization' against HIV-1 in certain infected individuals. Clearly, though, solving technical difficulties in the use of siRNAs to treat HIV-1-infected patients is also predicated on a suitable and efficient in vivo delivery system.

\section{Materials and methods}

\section{Materials}

Synthetic siRNA duplexes were designed and then chemically synthesized by Dharmacon Inc. (Lafayette, CO, USA). The 21-nucleotide RNA duplexes had twonucleotide deoxythymidine $3^{\prime}$ overhangs. The sequences of siRNA were as follows: CXCR4, 5'-GCA UGA CGG ACA AGU ACA G dTdT-3' (sense), 5'-CUG UAC UUG UCC GUC AUG C dTdT-3' (antisense); GFP 5'-GCA
GCA CGA CUU CUU CAA G dTdT-3' (sense), 5'-CUU GAA GAA GUC GUG CUG C dTdT-3' (antisense); APJ, 5'-GGU GCA GUG CUA CAU GGA C dTdT-3' (sense), 5'-GUC CAU GUA GCA CUG CAC C dTdT-3' (antisense). See Figure 5 for illustration of these siRNAs located on the homologous regions of the CXCR4, GFP

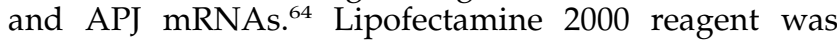
purchased from Invitrogen Inc. (Carlsbad, CA, USA) and Fugene 6 was purchased from Roche Inc. The full open reading frames of CXCR4 and APJ were amplified using PCR and subcloned in-frame into HindIII and BamHI sites of the pEGFP-N1 vector, to create GFP fusion constructs. ${ }^{64}$ All constructs were sequenced to confirm the correct sequence and orientation.

\section{Cell cultures and transfections}

HEK293 cells and the indicator human osteosarcoma cells, HOSCD4CXCR4 and HOSCD4CCR5 (AIDS Reagent

a Partial fold of CXCR4 mRNA used for siRNA selection Sense Primer (Red) Corresponds to CXCR4 mRVA region 300-318 |Accession HNM_003467]
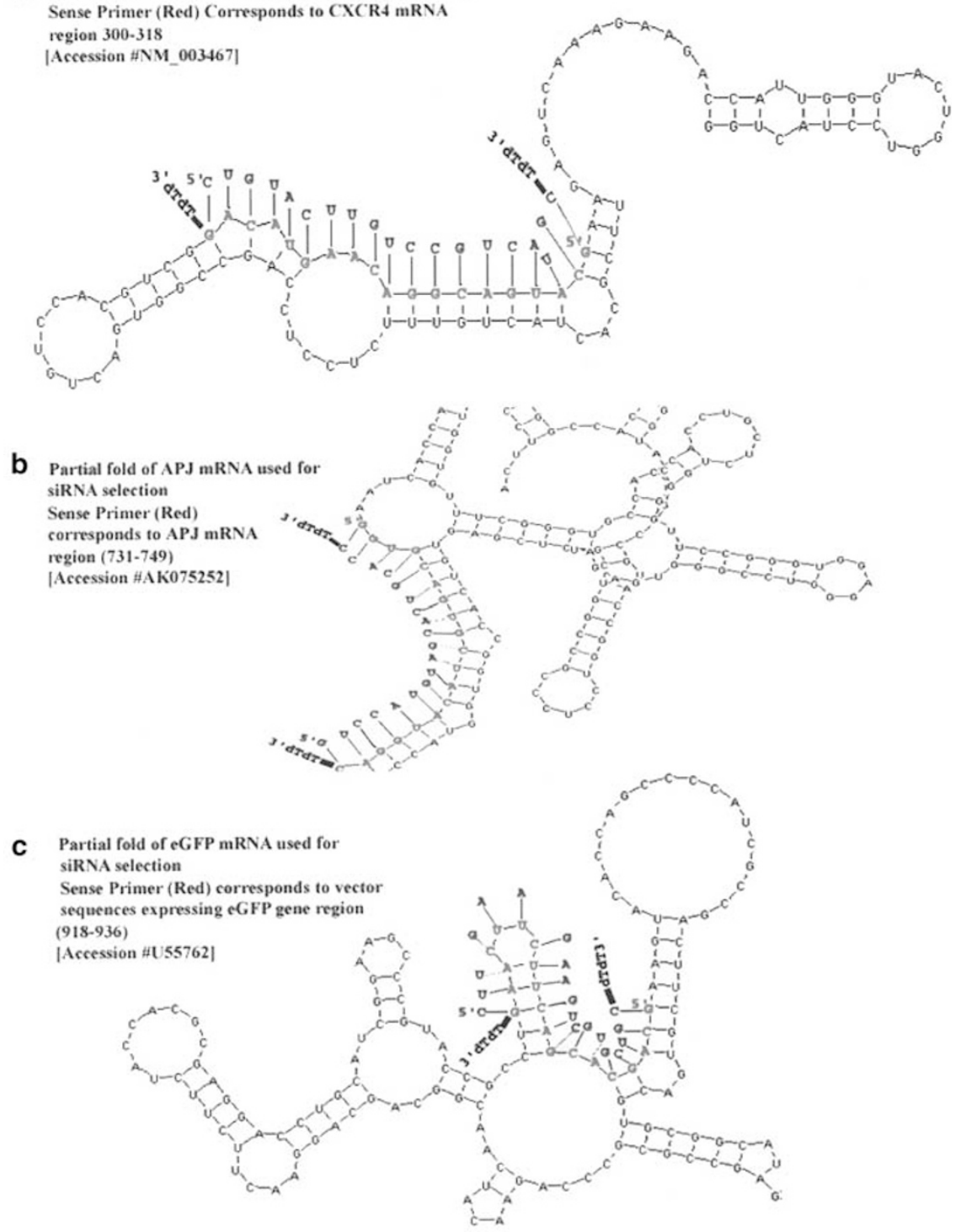

Figure 5 Location of siRNAs on homologous regions of CXCR4, APJ and GFP mRNAs. This schematic diagram illustrates the nucleotide homologies of the double-stranded siRNAs used in these studies for CXCR4, APJ and GFP. Genbank accession numbers for these target mRNAs are included. The sense strand is red and the antisense strand is blue in this diagram. Of note, $3^{\prime}$ dTdT overhangs are included on both strands. 
Repository, NIH), were maintained in DMEM supplemented with $10 \%$ fetal bovine serum (FBS) with $0.5 \mu \mathrm{g} /$ $\mathrm{ml}$ puromycin. HOSCD4CXCR4-GFP, HOSCD4APJ-GFP, 293-APJ-GFP and 293-CXCR4-GFP stable cell lines have been described previously. ${ }^{31,64}$ Cells were transfected at $90 \%$ confluence in six-well plates with plasmid DNA and siRNA ( $2 \mathrm{nM})$ by using Lipofectamine 2000 or Fugene 6 , according to the manufacturer's instructions. Cells were split after 1 day into Nunc two-well chamber slides for observation with fluorescence microscopy and in 24-well plates for HIV-1 infection assay. Fluorescence microscopy was performed on an Olympus System microscope, model BX60, with fluorescence attachment BX-FLA. ${ }^{66}$

\section{Fluorescence-activated cell sorting (FACS)}

siRNA-transfected cells and control cells were harvested $48 \mathrm{~h}$ post-transfection by washing twice with phosphatebuffered saline (PBS: $\mathrm{Ca}^{2+}$ and $\mathrm{Mg}^{2+}$ free) and detaching with $1 \mathrm{mM}$ EDTA containing PBS. The cells were further washed with PBS, and treated with initial and secondary antibodies (1:100 antibody in 3\% BSA/PBS) with incubation at $4{ }^{\circ} \mathrm{C}$ for $40 \mathrm{~min}$, sequentially. Antibodies used in flow cytometry were as follows: anti-human CXCR4 (Cat\#36190D), anti-human CCR5 (Cat\#36461A) and PE anti-mouse Igא (Cat\#559940) purchased from Pharmingen Inc. (San Diego, CA, USA). The cells were then analyzed in the flow cytometry core facility at Thomas Jefferson University.

\section{Gene reporter fusion assay}

A well-characterized gene reporter fusion assay was used to determine the coreceptor activity of CXCR4 in mediating HIV-1 viral entry, following a modified procedure published previously. ${ }^{70,71}$ Briefly, the effector 293 cells were infected with recombinant vaccinia virus expressing HIV-1 Env proteins from several viral strains and T7 RNA polymerase for $2 \mathrm{~h}$. Infected cells were then trypsinized, washed with PBS, resuspended in medium and incubated overnight at $32^{\circ} \mathrm{C}$ in the presence of rifampicin $(100 \mathrm{mg} / \mathrm{ml})$. HOSCD4CXCR4 cells as targets were cotransfected in six-well plates with plasmids encoding luciferase under control of the T7 promotor and CXCR4 siRNA, using the Lipofectamine 2000 reagent. At $4 \mathrm{~h}$ after transfection, cells were lifted, seeded in 24 -well plates and incubated at $37^{\circ} \mathrm{C}$ overnight. To initiate fusion, $10^{5}$ effector cells were added to each well and incubated at $37^{\circ} \mathrm{C}$. After $4 \mathrm{~h}$ of fusion, cells were lysed in $150 \mathrm{ml}$ of reporter lysis buffer (Pharmingen) and assayed for luciferase activity by using commercially available reagents (Pharmingen), with an FB12 Luminometer (Zylux Corporation, Maryville, TN, USA).

\section{HIV-1 infection assays}

To further determine anti-HIV-1 activity of siRNA against the coreceptor CXCR4, HOSCD4, HOSCD4CXCR4 and HOSCD4CCR5 cells (near-confluent cultures in six-well plates) were transfected with siRNA against CXCR4, and GFP as a control. At 2 days after transfection, the cells were infected with $10 \mathrm{ng}$ of p24 antigen equivalents of each virus strain overnight, and then thoroughly washed three times. The CXCR4-tropic virus, $\mathrm{NL}_{4-3}$, and the CCR5-tropic virus, ADA, were used in these studies. The supernatants were collected 3 days after infection for p24 antigen measurements using enzyme-linked immunosorbent assays (ELISA, Zeptometrix) ${ }^{40}$

HIV-1 proviral clones, expressing the luciferase (luc) gene in the nef open reading frame of HXB2 (X4-tropic) and ADA (R5-tropic) backbones, ${ }^{48}$ were used to produce viral stocks after transfection into 293 T cells. Normalized quantities of virus were used to infect U87-MG astrocytic-glial cells constitutively expressing CD4 alone, CD4 and CXCR4, or CD4 and CCR5 on their surfaces, $48 \mathrm{~h}$ after transfection of siRNA ( $2 \mathrm{nM})$. These cell lines were propagated in DMEM with $10 \%$ FBS and $1 \mu \mathrm{g} / \mathrm{ml}$ puromycin. At $24 \mathrm{~h}$ after viral infection, cells $\left(2-8 \times 10^{5}\right)$ were lysed in reporter lysis buffer and assayed for luciferase activity, as per the manufacturer's instructions (Pharmingen), in a luminometer. ${ }^{31}$

\section{Acknowledgements}

We thank Dr B Cullen (Duke University) for kindly providing the luciferase-reported HIV-1 proviral clones and Ms Rita M Victor and Ms Brenda O Gordon for excellent secretarial assistance. The U87MG reporter cell line was obtained from the AIDS Reagent Repository (NIH), submitted by Drs Deng and D Littman. This work was supported in part by USPHS Grants NS27405, MH58526, NS44513 and NS41864 to RJP.

\section{References}

1 Caplen NJ et al. Specific inhibition of gene expression by small double-stranded RNAs in invertebrate and vertebrate systems. Proc Natl Acad Sci USA 2001; 98: 9742-9747.

2 Elbashir SM et al. Duplexes of 21-nucleotide RNAs mediate RNA interference in cultured mammalian cells. Nature 2001; 411: 494-498.

3 Elbashir SM, Lendeckel W, Tuschl T. RNA interference is mediated by 21- and 22-nucleotide RNAs. Genes Dev 2001; 15: 188-200.

4 Fire A et al. Potent and specific genetic interference by doublestranded RNA in Caenorhabditis elegans. Nature 1998; 391: 806-811.

5 Wianny F, Zernicka-Goetz M. Specific interference with gene function by double-stranded RNA in early mouse development. Nat Cell Biol 2000; 2: 70-75.

6 Choe $\mathrm{H}$ et al. The orphan seven-transmembrane receptor apj supports the entry of primary T-cell-line-tropic and dualtropic human immunodeficiency virus type 1. J Virol 1998; 72: 6113-6118.

7 Mattick JS. Non-coding RNAs: the architects of eukaryotic complexity. EMBO Rep 2001; 2: 986-991.

8 Storz G. An expanding universe of noncoding RNAs. Science 2002; 296: 1260-1263.

9 Ahlquist P. RNA-dependent RNA polymerases, viruses, and RNA silencing. Science 2002; 296: 1270-1273.

10 Kitabwalla M, Ruprecht RM. RNA interference - a new weapon against HIV and beyond. N Engl J Med 2002; 347: 1364-1367.

11 Llave C, Kasschau KD, Carrington JC. Virus-encoded suppressor of posttranscriptional gene silencing targets a maintenance step in the silencing pathway. Proc Natl Acad Sci USA 2000; 97: 13401-13406.

12 Paul CP, Good PD, Winer I, Engelke DR. Effective expression of small interfering RNA in human cells. Nat Biotechnol 2002; 29: 505-508.

13 Pomerantz RJ. RNA interference meets HIV-1: will silence be golden? Nat Med 2002; 8: 659-660. 
14 Sharp PA. RNA interference - 2001. Genes Dev 2001; 15: 485-490.

15 Song $\mathrm{E}$ et al. RNA interference targeting Fas protects mice from fulminant hepatitis. Nat Med 2003; 9: 347-351.

16 Voinnet $\mathrm{O}$, Lederer $\mathrm{C}$, Baulcombe DC. A viral movement protein prevents spread of the gene silencing signal in Nicotiana benthamiana. Cell 2000; 103: 157-167.

17 Waterhouse PM, Wang MB, Lough T. Gene silencing as an adaptive defence against viruses. Nature 2001; 411: 834-842.

18 Yu JY, DeRuiter SL, Turner DL. RNA interference by expression of short-interfering RNAs and hairpin RNAs in mammalian cells. Proc Natl Acad Sci USA 2002; 99: 6047-6052.

19 Lipardi C, Wei Q, Paterson BM. RNAi as random degradative PCR: siRNA primers convert mRNA into dsRNAs that are degraded to generate new siRNAs. Cell 2001; 107: 297-307.

20 Sijen $\mathrm{T}$ et al. On the role of RNA amplification in dsRNAtriggered gene silencing. Cell 2001; 107: 465-476.

21 Schwarz DS, Hutvagner G, Haley B, Zamore PD. Evidence that siRNAs function as guides, not primers, in the Drosophila and human RNAi pathways. Mol Cell 2002; 10: 537-548.

22 Miyagishi M, Taira K. U6 promoter-driven siRNAs with four uridine $3^{\prime}$ overhangs efficiently suppress targeted gene expression in mammalian cells. Nat Biotechnol 2002; 20: 497-500.

$23 \mathrm{Li} \mathrm{H}, \mathrm{Li} \mathrm{WX}$, Ding SW. Induction and suppression of RNA silencing by an animal virus. Science 2002; 296: 1319-1321.

24 Dornburg R, Pomrantz RJ. Gene therapy and HIV-1 infection: experimental approaches, shortcomings and possible solutions. In: Friedman H, Bendinelli M (eds) Human Retroviral Infections: Immunological and Molecular Theories. Plenum Publishers: New York, 2000, pp 307-323.

25 Murphy PM. The molecular biology of leukocyte chemoattractant receptors. Annu Rev Immunol 1994; 12: 593-633.

26 Zhang $\mathrm{L}$ et al. Chemokine coreceptor usage by diverse primary isolates of human immunodeficiency virus type 1. J Virol 1998; 72: 9307-9312.

27 Bleul CC et al. The lymphocyte chemoattractant SDF-1 is a ligand for LESTR/fusin and blocks HIV-1 entry. Nature 1996; 382: 829-833.

28 Doranz BJ et al. A dual-tropic primary HIV-1 isolate that uses fusin and the beta-chemokine receptors CKR-5, CKR-3, and CKR-2b as fusion cofactors. Cell 1996; 85: 1149-1158.

29 Feng Y, Broder CC, Kennedy PE, Berger EA. HIV-1 entry cofactor: functional cDNA cloning of a seven-transmembrane, $\mathrm{G}$ protein-coupled receptor. Science 1996; 272: 872-877.

30 Oberlin E et al. The CXC chemokine SDF-1 is the ligand for LESTR/fusin and prevents infection by T-cell-line-adapted HIV1. Nature 1996; 382: 833-835.

31 Zhou N et al. Structural and functional characterization of human CXCR4 as a chemokine receptor and HIV-1 co-receptor by mutagenesis and molecular modeling studies. J Biol Chem 2001; 276: 42826-42833.

$32 \mathrm{Ma} \mathrm{Q}$ et al. Impaired B-lymphopoiesis, myelopoiesis, and derailed cerebellar neuron migration in CXCR4- and SDF-1deficient mice. Proc Natl Acad Sci USA 1998; 95: 9448-9453.

33 Nagasawa $\mathrm{T}$ et al. Defects of B-cell lymphopoiesis and bonemarrow myelopoiesis in mice lacking the CXC chemokine PBSF/SDF-1. Nature 1996; 382: 635-638.

34 Zou YR et al. Function of the chemokine receptor CXCR4 in haematopoiesis and in cerebellar development. Nature 1998; 393: 595-599.

35 Scarlatti G et al. In vivo evolution of HIV-1 co-receptor usage and sensitivity to chemokine-mediated suppression. Nat Med 1997; 3: 1259-1265.

36 Penn ML et al. CXCR4 utilization is sufficient to trigger CD4+ T cell depletion in HIV-1-infected human lymphoid tissue. Proc Natl Acad Sci USA 1999; 96: 663-668.

37 Schramm B et al. Viral entry through CXCR4 is a pathogenic factor and therapeutic target in human immunodeficiency virus type 1 disease. J Virol 2000; 74: 184-192.
38 Zhou N et al. Binding of ALX40-4C to APJ, a CNS-based receptor, inhibits its utilization as a co-receptor by HIV-1. Virology 2003; 312: 196-203.

39 Fujii N, Tamamura H. Peptide-lead CXCR4 antagonists with high anti-HIV activity. Curr Opin Investig Drugs 2001; 2: 1198-1202.

40 BouHamdan $\mathrm{M}$ et al. Inhibition of HIV-1 infection by downregulation of the CXCR4 co-receptor using an intracellular single chain variable fragment against CXCR4. Gene Therapy 2001; 8: 408-418.

41 Chen JD et al. Inactivation of HIV-1 chemokine co-receptor CXCR-4 by a novel intrakine strategy. Nat Med 1997; 3: 1110-1116.

42 Doranz BJ et al. Safe use of the CXCR4 inhibitor ALX40-4C in humans. AIDS Res Hum Retroviruses 2001; 17: 475-486.

$43 \mathrm{Ge} \mathrm{Q}$ et al. RNA interference of influenza virus production by directly targeting mRNA for degradation and indirectly inhibiting all viral RNA transcription. Proc Natl Acad Sci USA 2003; 100: 2718-2723.

44 Gitlin L, Karelsky S, Andino R. Short interfering RNA confers intracellular antiviral immunity in human cells. Nature 2002; 418: 430-434.

45 Seo MY, Abrignani S, Houghton M, Han JH. Small interfering RNA-mediated inhibition of hepatitis $C$ virus replication in the human hepatoma cell line Huh-7. J Virol 2003; 77: 810-812.

46 Wilson JA et al. RNA interference blocks gene expression and RNA synthesis from hepatitis $C$ replicons propagated in human liver cells. Proc Natl Acad Sci USA 2003; 100: 2783-2788.

47 Capodici J, Kariko K, Weissman D. Inhibition of HIV-1 infection by small interfering RNA-mediated RNA interference. J Immunol 2002; 169: 5196-5201.

48 Coburn GA, Cullen BR. Potent and specific inhibition of human immunodeficiency virus type 1 replication by RNA interference. J Virol 2002; 76: 9225-9231.

49 Jacque JM, Triques K, Stevenson M. Modulation of HIV-1 replication by RNA interference. Nature 2002; 418: 435-438.

50 Lee NS et al. Expression of small interfering RNAs targeted against HIV-1 rev transcripts in human cells. Nat Biotechnol 2002; 20: $500-505$.

51 Novina CD et al. siRNA-directed inhibition of HIV-1 infection. Nat Med 2002; 8: 681-686.

52 Surabhi RM, Gaynor RB. RNA interference directed against viral and cellular targets inhibits human immunodeficiency Virus Type 1 replication. J Virol 2002; 76: 12963-12973.

53 Qin XF, An DS, Chen IS, Baltimore D. Inhibiting HIV-1 infection in human $\mathrm{T}$ cells by lentiviral-mediated delivery of small interfering RNA against CCR5. Proc Natl Acad Sci USA 2003; 100: 183-188.

54 Doranz BJ et al. A small-molecule inhibitor directed against the chemokine receptor CXCR4 prevents its use as an HIV-1 coreceptor. J Exp Med 1997; 186: 1395-1400.

55 Kusunoki A et al. Antisense oligodeoxynucleotide complementary to CXCR4 mRNA block replication of HIV-1 in COS cells. Nucleosides Nucleotides 1999; 18: 1705-1708.

56 McKnight A et al. Inhibition of human immunodeficiency virus fusion by a monoclonal antibody to a coreceptor (CXCR4) is both cell type and virus strain dependent. J Virol 1997; 71: 1692-1696.

57 Murakami Tet al. A small molecule CXCR4 inhibitor that blocks T cell line-tropic HIV-1 infection. J Exp Med 1997; 186: 1389-1393.

58 O'Brien WA et al. Anti-human immunodeficiency virus type 1 activity of an oligocationic compound mediated via gp120 V3 interactions. J Virol 1996; 70: 2825-2831.

59 Schols D et al. Inhibition of T-tropic HIV strains by selective antagonization of the chemokine receptor CXCR4. J Exp Med 1997; 186: 1383-1388.

60 Tamamura $\mathrm{H}$ et al. Development of specific CXCR4 inhibitors possessing high selectivity indexes as well as complete stability 
in serum based on an anti-HIV peptide T140. Bioorg Med Chem Lett 2001; 11: 1897-1902.

61 Edinger AL et al. An orphan seven-transmembrane domain receptor expressed widely in the brain functions as a coreceptor for human immunodeficiency virus type 1 and simian immunodeficiency virus. J Virol 1998; 72: 7934-7940.

62 Hosoya $\mathrm{M}$ et al. Molecular and functional characteristics of APJ. Tissue distribution of mRNA and interaction with the endogenous ligand apelin. J Biol Chem 2000; 275: 21061-21067.

63 Cayabyab $M$ et al. Apelin, the natural ligand of the orphan seven-transmembrane receptor APJ, inhibits human immunodeficiency virus type 1 entry. J Virol 2000; 74: 11972-11976.

64 Zhou N et al. Cell-cell fusion and internalization of the CNSbased, HIV-1 co-receptor, APJ. Virology 2003; 307: 22-36.

65 Gabuzda D, He J, Ohagen A, Vallat AV. Chemokine receptors in HIV-1 infection of the central nervous system. Semin Immunol 1998; 10: 203-213.

66 Mukhtar M et al. Primary isolated human brain microvascular endothelial cells express diverse HIV/SIV-associated chemokine coreceptors and DC-SIGN and L-SIGN. Virology 2002; 297: 78-88.

67 Hesselgesser $\mathrm{J}$ et al. CD4-independent association between HIV-1 gp120 and CXCR4: functional chemokine receptors are expressed in human neurons. Curr Biol 1997; 7: 112-121.

68 Krichevsky AM, Kosik KS. RNAi functions in cultured mammalian neurons. Proc Natl Acad Sci USA 2002; 99: 11926-11929.

69 Martinez MA et al. Suppression of chemokine receptor expression by RNA interference allows for inhibition of HIV-1 replication. Aids 2002; 16: 2385-2390.

70 Rucker J et al. Cell-cell fusion assay to study role of chemokine receptors in human immunodeficiency virus type 1 entry. Methods Enzymol 1997; 288: 118-133.

71 Nussbaum O, Broder CC, Berger EA. Fusogenic mechanisms of enveloped-virus glycoproteins analyzed by a novel recombinant vaccinia virus-based assay quantitating cell fusion-dependent reporter gene activation. J Virol 1994; 68: 5411-5422. 